\title{
Temperature field simulations during selective laser melting process based on fully threaded tree
}

\author{
Wei Duan, *Ya-jun Yin and Jian-xin Zhou \\ State Key Laboratory of Materials Processing and Die \& Mould Technology, Huazhong University of Science and Technology, Wuhan 430074, \\ China
}

\begin{abstract}
Selective laser melting (SLM) is a promising technique for additive manufacturing. During SLM of metallic powder, the temperature field and thermal history are important to understand physical phenomena involved. The purpose of this study is to simulate the temperature field during the SLM process of a hollow cylinder shape part based on a fully threaded tree (FTT) technique, and to analyze the temperature variation with time in different regions of the part. A revised model for temperature field simulation in the SLM process was employed, which includes the transition of powder-to-dense sub-model and a moving volumetric Gaussian distribution heat source sub-model. The FTT technique is then adopted as an adaptive mesh strategy in the simulation. The simulation result shows that during the SLM process of cylinder part, the temperature of inner powder bed is obviously higher than external one. The temperature at the internal of the molten pool is also higher than external, which may lead to differences in microstructures and other properties between the two regions.
\end{abstract}

Key words: fully threaded tree; selective laser melting; temperature distribution; numerical simulation
CLC numbers: TP391.99
Document code: $\mathrm{A}$
Article ID: 1672-6421(2017)05-405-07

\begin{abstract}
Celective laser melting (SLM) is the commonly term $\checkmark$ to describe the process in which a high energy laser beam scans the surface of a metal powder bed and the melted powder solidifies to form the bulk part ${ }^{[1]}$. SLM make it possible to create final function parts without post processing steps. Manufacturing businesses aiming to deliver their new products more quickly increasingly employ selective laser sintering/melting (SLS/SLM) for fabricating high quality, low cost, repeatable, and reliable alloy powdered parts for automotive, aerospace, and aircraft applications ${ }^{[2]}$. SLS can indirectly form integral control-shaped capsule for hot isostatic pressing (HIP) and mould core for casting, and SLM can directly and rapidly form HIP-shaped capsule ${ }^{[3]}$.

SLM is complicated because of its high laser scan velocity and phase transformation in a sharply short time. Temperature field in this process is inhomogeneous ${ }^{[4-6]}$. Meanwhile, temperature evolution history would have significant
\end{abstract}

\footnotetext{
*Ya-jun Yin
}

Male, born in 1985, Ph.D. His research is mainly on simulation of solidification microstructure evolution, additive manufacturing and hot isostatic pressing.

E-mail: yinyajun436@hust.edu.cn.

Received: 2017-08-15; Accepted: 2017-09-21 effects on final quality of parts. Large temperature gradients increase residual stresses and distortion and even lead to cracks in parts. Therefore, it is significant for SLM researchers to understand the mechanism of this process and effects of the parameters.

Experiments regarding temperature fields, which greatly influence the formation of defects during the process, are lacking. It is a difficult work for most researchers to gain the detailed temperature field and its history during SLM process based on present experiment conditions. So, numerical simulation plays a significant role in the study of thermal behaviour during SLM process. The aim to perform temperature field simulation of SLM processes is to reduce the experimental cost, reveal the transient thermal history that cannot be obtained by limited experiment, and understand the evolution of microstructure and residual stress ultimately.

Simulations regarding temperature evolution have been conducted. Zäh and Lutzmann ${ }^{[7]}$ created a detailed thermal model to develop the knowledge of adequate parameter settings that are dependent on the material used. Kuchi ${ }^{[8]}$ used parametric finite element modelling with Abaqus to investigate the effects of 
finite geometry and associated non-steady state changes in process variables, such as beam power and scan velocity, on the thermal conditions controlling microstructure. Some researchers have also studied temperature field during a similar additive manufacturing (AM) process. Sun et al. proposed a three-dimensional finite model to simulate the temperature field in selective laser melting ${ }^{[9]}$.

However, the previous simulation studies were mainly concerned with the relationship between the transient response of the melt pool dimensions and microstructure control with process variables, such as rapid changes in beam power and travel velocity ${ }^{[10,11]}$. Additionally, most simulations are restricted to two dimensions, and, in some simulations, the deposit is processed with fewer layers due to the large amount of calculations required, which increases the limitation in application for industrial large and complex components. Thus, 3D temperature simulations are imperative and desirable.

The purposes of present work are to apply a fully threaded tree (FTT) technique to 3D simulation of temperature field during the SLM process and to make grids change and melt pool characteristic analysis based on 3D temperature field. The present study introduces the mathematical-physical model of SLM process, and successfully uses the FTT adaptive grid technique to simulate the temperature field in the SLM process. Melt pool is also depicted and analyzed in a certain cross section.

\section{Physical model of SLM}

\subsection{Assumptions}

Figure 1 is a schematic representation of heat transfer in SLM process ${ }^{[12]}$. The laser scans on the top of the powder bed follow a prescribed scan pattern. The heat transfer process consists of powder bed radiation, convection between the powder bed and environment, and heat conduction inside the powder bed and between the powder bed and substrate.

Due to the complex nature of SLM mentioned above, following assumptions are made in this study to simplify the model:

(1) The powder bed is considered to be a continuous material medium;

(2) The flow of molten metal in the molten pool is not

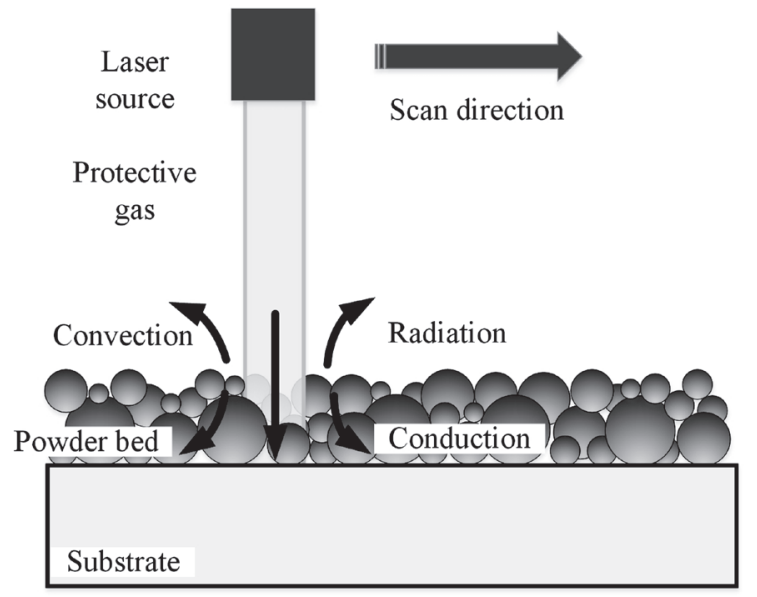

considered;

(3) The thermal physical properties of materials, such as thermal conductivity, are considered to be varied with temperature;

(4) To simplify the calculation, the latent heat of phase change, metal gasification and the powder layer shrinkage are ignored.

(5) The volumetric heat resource is treated as an internal heat generation.

\subsection{Model}

Heat conduction is depicted in Eq. (1) according to the Fourier Law:

$$
\rho \mathrm{C}_{p}\left(\frac{\partial T}{\partial t}\right)=\nabla \cdot(\nabla \lambda T)+Q
$$

where $T$ is temperature, $t$ is time, $C_{p}$ is specific heat capacity, $\rho$ is density, $\lambda$ is the heat conductivity of the material. It is assumed that the physical parameters of the material, $C_{p}, \rho$, and $\lambda$ are independent of temperature. $Q$ represents the heat source given by the model of the SLM process.

Proper boundary conditions must be set to obtain the more accurate simulation results. The heat radiation is considered, and can be expressed as Eq. (2):

$$
k \frac{\partial T}{\partial \vec{n}}=-\varepsilon_{r} \sigma\left(T^{4}-T_{\infty}^{4}\right)
$$

where $\sigma$ is the Stefan-Boltzmann constant $\left(\sigma=5.67 \times 10^{-8}\right.$ $\mathrm{W} \cdot \mathrm{m}^{-2} \cdot \mathrm{K}^{-4)}, \varepsilon_{r}$ is the Blackbody radiation coefficient, $k$ is the coefficient of heat transfer, and $T_{\infty}$ is the ambient temperature.

The heat convection is also considered, and can be expressed as Eq. (3):

$$
k \frac{\partial T}{\partial \vec{n}}=-h_{\text {conv }}\left(T-T_{\infty}\right)
$$

where $h_{\text {conv }}$ is the convection heat transfer coefficient.

The laser energy flux could be loaded in Gaussian face heat source or Gaussian volumetric heat source ${ }^{[13]}$. When the laser interacts with solid metal, the depth of laser penetration is less than $1 \mathrm{~mm}$, thus Gaussian face heat source could be adopted. However, when laser interacts with porous material such as metal powder bed, the depth of penetration could not be ignored, thus Gaussian volumetric heat source will be adopted. For this work, the laser energy density follows a Gaussian profile on the $x$ and $y$ coordinates and an exponential decay profile on the $z$ coordinate ${ }^{[14]}$ :

$$
q(r, z)=\frac{2 A P}{\pi R^{2} H} \exp \left(-\frac{r^{2}}{R^{2}}\right) \exp \left(-\frac{|z|}{H}\right)
$$

where $A$ is the heat absorptivity of laser beam on the metal powder bed, $P$ is the laser power, $R$ is the radius corresponding to the point where the laser irradiation diminishes to $1 / \mathrm{e}^{2}$ of its maximum value, and $H$ is the penetration depth of the laser in powder bed.

There are three kinds of undetermined physical variables consisting of material thermophysical properties, laser technical parameters and boundary condition parameters. 


\section{Material thermal physical properties}

During the SLM process, the state of material involves powder, liquid and solid, and the properties of them changed with temperature. Thermal physical properties determine to a great extent the temperature field of the formed part. In this study, the utilized powder and the substrate material are both TC4 alloy.

\subsection{Thermal conductivity}

The thermal conductivity of powder bed differs greatly with dense solid materials due to their incompact structure. It leads to the complex heat transfer mechanism of powder bed as there are convection and radiation of fluid inside the voids in addition to powder-solid heat conduction. The effective conductivity calculation equation of the powder bed was proposed by Sakase Yagi and Daizo Kunii ${ }^{[15]}$, and is shown as following:

$$
k_{\text {powder }}=\frac{\beta k_{\mathrm{s}}(1-\varphi)}{1+\xi \frac{k_{\mathrm{s}}}{k_{\mathrm{g}}}}
$$

where $k_{\text {powder }}$ is effective thermal conductivity of the powder bed, $\beta$ is the ratio of effective length between the center to average diameter of the powder particles, $k_{\mathrm{s}}$ is the thermal conductivity of the solid, $k_{\mathrm{g}}$ is thermal conductivity of gas surrounding the powder particles, $\varphi$ is the porosity of powder bed, and $\xi$ can be empirically taken as $0.02 \times 10^{2(\varphi-0.3)[16]}$.

Based on Yagi-Kunii model ${ }^{[15]}$, an empirical modification calculation equation was proposed as following ${ }^{[16]}$ :

$$
k_{\text {powder }}=\left(6.3+22 \sqrt{0.06 k_{\mathrm{s}}-0.016}\right) \times \frac{k_{\mathrm{s}}(1-\varphi)}{\frac{k_{\mathrm{s}}}{k_{\mathrm{g}}} 10^{0.523-0.594 \varphi}-1}
$$

The heat transfer mechanism of gas is that the gas molecular thermodynamic movement and collisions between gas molecular lead to heat transfer. The thermal conductivity of the gas was calculated by empirical equation ${ }^{[17]}$ :

$$
k_{\mathrm{g}}=\left(5.86 \times 10^{-5}+1.7639 \times 10^{-7} T\right) \times 418.4
$$

The solid thermal conductivity of TC4 was listed in Table $1^{[18]}$. Thermal conductivity of solid TC4 was described by Eq. (8) fitted in a straight line by data from Table 1.

$$
k_{\mathrm{s}}=1.83+0.017 T
$$

In comparison to the thermal conduction problem of solid material, during SLM process, the sintered sample varies from the powder state to the dense state, and the thermal conductivity changes depending on the porosity and temperature of the powder bed. At the initial stage, the powder bed is initially in powder state, and the thermal conductivity of the powder bed is mainly determined by the porosity, particle diameter, emissivity, and temperature according to equation (5) to (8). During the laser heating stage, once the temperature is higher than the sintering temperature, the powder-to-dense transition will take effect during the high temperature portion and then the powders convert to the middle state irreversibly. Once the temperature is higher than the melting point, the powders convert to the dense molten metal. It is assumed that the relationship between thermal conductivity of middle state material and temperature is linear, during the powder-to-dense process. Therefore, the heat conductivity is expressed in piecewise function of $T$ as:

$$
k_{\mathrm{c}}=\left\{\begin{array}{l}
k_{\mathrm{powder}}, T_{0}<T<T_{\mathrm{s}} \\
\frac{k_{\mathrm{s}}\left(T_{m}\right)-k_{\text {powder }}\left(T_{\mathrm{s}}\right)}{T_{\mathrm{m}}-T_{\mathrm{s}}} T+k_{\text {powder }}\left(T_{\mathrm{s}}\right), T_{\mathrm{s}}<T<T_{\mathrm{m}} \\
k_{\mathrm{s}}, T>T_{\mathrm{m}}
\end{array}\right.
$$

where $k_{\mathrm{e}}$ is the effective conductivity of material during SLM process, $T_{\mathrm{s}}$ is defined as initial sintering temperature and $T_{\mathrm{m}}$ is melting temperature.

During the cooling stage, the thermal conductivity of the dense phase is only treated as a function of temperature since the liquid phase and solid phase are fully dense.

\subsection{Other properties}

For solid material, density, $\rho_{\mathrm{s}}$, is the function of temperature. For powder material, the relationship between porosity of powder bed, $\varphi$, and apparent density, $\rho_{0}$, is described as:

$$
\varphi=\frac{\rho_{\mathrm{s}}-\rho_{0}}{\rho_{\mathrm{s}}}
$$

It is assumed that the relationship between density of middle state material and temperature is linear, during the powder-to-

Table 1: Physical properties for TC4 at various temperatures

$\begin{array}{cccc}\text { Temperature, } T\left({ }^{\circ} \mathrm{C}\right) & \text { Specific heat capacity }\left(\mathrm{J} \cdot \mathrm{Kg}^{-1} \cdot{ }^{\circ} \mathrm{C}^{-1}\right) & \text { Thermal conductivity }\left(\mathbf{W} \cdot \mathrm{m}^{-1} \cdot{ }^{\circ} \mathbf{C}^{-1}\right) & \text { Density }\left(\mathbf{g} \cdot \mathrm{cm}^{-3}\right) \\ 20 & 611 & 6.8 & 4.44 \\ 100 & 624 & 7.4 & - \\ 200 & 653 & 8.7 & - \\ 300 & 674 & 9.8 & - \\ 400 & 691 & 10.3 & - \\ 500 & 703 & 11.8 & - \\ 600 & 710 & 12.8 & - \\ 700 & 712 & 13.5 & \end{array}$


dense process. Therefore, the density is expressed in piecewise function of $T$ as:

$$
\rho=\left\{\begin{array}{l}
\rho_{0}, T_{0}<T<T_{\mathrm{s}} \\
\frac{\rho_{\mathrm{s}}\left(T_{\mathrm{m}}\right)-\rho_{0}\left(T_{\mathrm{s}}\right)}{T_{\mathrm{m}}-T_{\mathrm{s}}} T+\rho_{0}\left(T_{\mathrm{s}}\right), T_{\mathrm{s}}<T<T_{\mathrm{m}} \\
\rho_{\mathrm{s}}, T>T_{\mathrm{m}}
\end{array}\right.
$$

Since the density of protective gas among metal powder particles is rather small, the mixture specific heat can be calculated as following ${ }^{[19]}$ :

$$
C_{\text {mix }}=\frac{\rho_{1} C_{1} \zeta_{1}+\rho_{2} C_{2} \zeta_{2}}{\rho_{1} \zeta_{1}+\rho_{2} \zeta_{2}}=\frac{\rho_{1} C_{1} \zeta_{1}}{\rho_{1} \zeta_{1}}=C_{1}
$$

where $C_{1}$ is the specific heat of powder particle, $C_{2}$ is the specific heat of protective gas among powder, $\rho_{1}$ and $\rho_{2}$ are densities of metal powder particle and gas respectively, and $\xi_{1}$ and $\xi_{2}$ are volume percentage of metal powder particle and gas, respectively.

\section{Finite difference model}

\subsection{Process parameters}

This analysis was carried out on a three-dimensional meshed geometry model which is a representation of the physical mapping of the sintered sample and substrate. The dimensions of the powder bed are $40 \mu \mathrm{m}$ in height and 2,560 $\mu \mathrm{m}$ in both length and width, while the substrate has a width and length of 2,560 $\mu \mathrm{m}$ and height of $40 \mu \mathrm{m}$. The calculation domain is $2,560 \times 2,560 \times 2,560 \mu^{3}$ and the scan path is a circle whose diameter is $1280 \mu \mathrm{m}$. In the scanning process of a track, the movement heat source was considered to occur in discrete time steps, and each time period is decided by the element size and laser flight speed; moreover, the laser beam moves stepwise by one element in the scan path. The stepping sequence begins at the starting spot and continues. Table 2 summarizes the parameters used in the finite difference model.

\subsection{FTT grids model}

FTT is a kind of tree structure used to describe threedimensional space. Each node of FTT represents a cube volume element and has eight child nodes standing for eight small cubes. The total volume of eight child cubes equals to the volume of their farther cube, and the center nodes of child cubes are bifurcated from the center node of their farther. As shown in Fig. 2, different colors refer to deferent cubes in the left figure, and the sketch map in right illustrates the logical relationship. The original cube is defined as the root cube and the next level of cubes bifurcated from it are defined as child cubes. Any cube with child cubes, no matter it is a root cube or not, is termed as farther cube. On the contrary, any region without child cubes is termed as leaf cube. All cubic grids are logically organized in the FTT structure within the entire computational domain. The size of cube is $L$ and is subdivided into a number of cubic grids of various sizes, including $1 / 2,1 / 4,1 / 8$, etc., of $L$.
Table 2: Computational parameters used for simulation

\begin{tabular}{lcc}
\multicolumn{1}{c}{ Parameters } & Symbol & Value \\
Laser power $(\mathrm{W})$ & $P$ & 110 \\
Laser spot radius $(\mu \mathrm{m})$ & $R$ & 100 \\
Scan speed $\left(\mathrm{mm} \cdot \mathrm{s}^{-1}\right)$ & $V$ & 20 \\
Powder porosity & $\varphi$ & 0.5 \\
Average particle size $(\mu \mathrm{m})$ & $D$ & 20 \\
Powder bed thickness $(\mu \mathrm{m})$ & $d$ & 100 \\
Optical penetration depth in powder $(\mu \mathrm{m})$ & $H$ & 100 \\
Ambient temperature $\left({ }^{\circ} \mathrm{C}\right)$ & $T_{\infty}$ & 25 \\
Initial temperature $\left({ }^{\circ} \mathrm{C}\right)$ & $T_{0}$ & 25
\end{tabular}

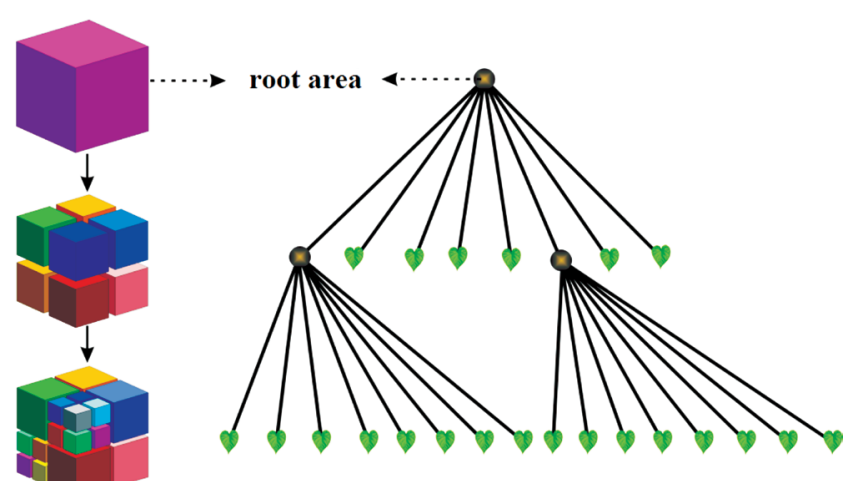

Fig. 2: FTT example

Each father cubic grid in FTT has 8 child cubic grids. Each child grid could be a father grid itself, as it splits into 8 pieces. The layer number of the grid is an important parameter, and it represents the degree of the grids refinement or coarsening. During SLM, as shown in Fig. 3, as the laser spot arrives at a certain region, the grids split and the layer numbers of these grids increase correspondingly, because the temperature of these grids achieves and exceeds a certain value as a criterion. This process is defined as grids refining. However, as laser spot leaves and temperature decreases, the grids merge and the layer number of certain region decreases correspondingly. In contrast, this process is defined as grids coarsening. In addition to temperature, temperature gradient is also a criterion for grid coarsening and refining.

Derivative in mathematical model is treated in the finite difference method. There are some differences in calculation methods of neighbor grids' value between FTT and other gird technique. There are three different types of neighbor grids relationship, and three types of calculate methods, respectively. More details were involved in the previous research ${ }^{[20]}$.

\section{Results and discussion}

\subsection{Grids changing in temperature field}

In present study, grids were classified into 10 layers. As mentioned above, layer is an important notion represents 

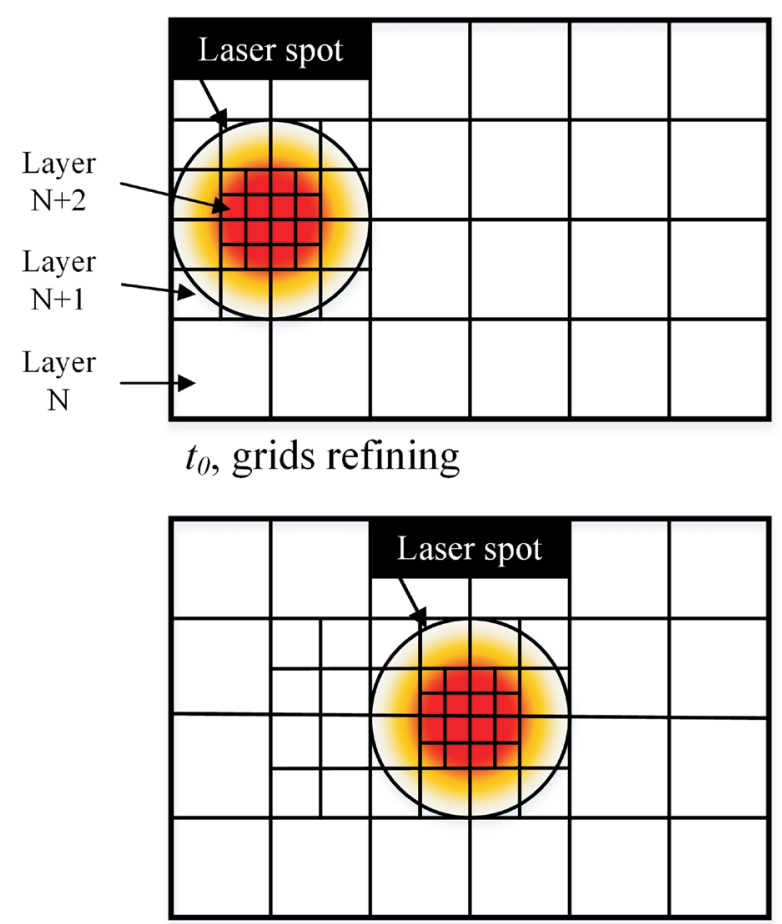

$t_{1}$, grids refining and coarsening

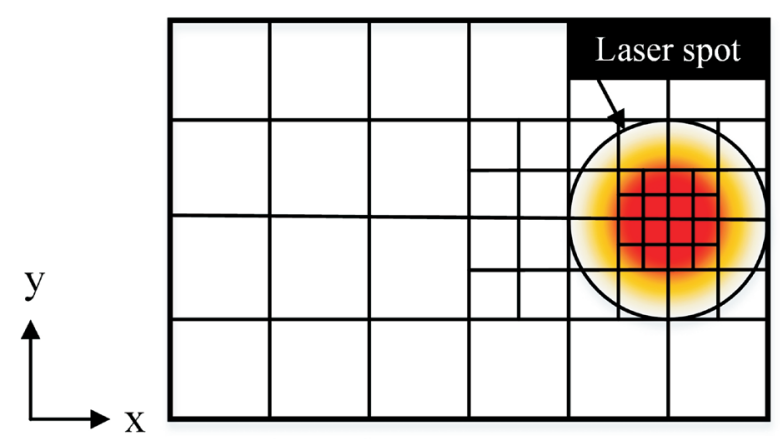

$t_{2}$, grids refining and coarsening

Fig. 3: Grids refining and coarsening in FTT

the level of grid refinement and coarsening. All the grids are listed in Table 3. As shown in Fig. 4, layer 0 is the calculation domain, a cube about $5,120 \mu \mathrm{m}$ on a side. Layer 6 is the middle layer of the grids without coarsening or refinement. Furthermore, layer 4 is the maximum level to which the grids allow to be coarsened, and layer 9 is the minimum level to which the grids can be refined.

In Fig. 5, the temperature field plots of the SLM process at three different moments are adopted to illustrate the changes of meshes. It can be seen that the central part of the laser beam refines due to the high temperature, which is coincident with the adaptive refinement model mentioned in above section. The smaller grids provide more precise temperature value and are favorable for some other coupled fields simulations in the future work. Furthermore, it can be seen from Fig. 5 that peripheries of the heat source are also refined due to the high temperature gradient, since temperature gradient as a criterion is added into the refined model. Once the temperature gradient of a region achieves a set value, the current grids will then be refined. However, although finer grid leads to higher
Table 3: Grids level and grid size

$\begin{array}{ccc}\text { Layer of grids } & \begin{array}{c}\text { Length of } \\ \text { side }(\boldsymbol{\mu m})\end{array} & \text { Comments } \\ 0 & 5,120 & \text { The calculation domain } \\ 1 & 2,560 & \\ 2 & 1,280 & \\ 3 & 640 & \text { The maximum layer to which the grids } \\ 4 & 320 & \text { can be coarsened. } \\ 5 & 160 & \text { The middle layer of the grids without } \\ 6 & 80 & \text { coarsening or refinement. } \\ 7 & 40 & \text { The minimum layer to which the grids } \\ 8 & 20 & \text { can be refined. } \\ 9 & 10 & \end{array}$

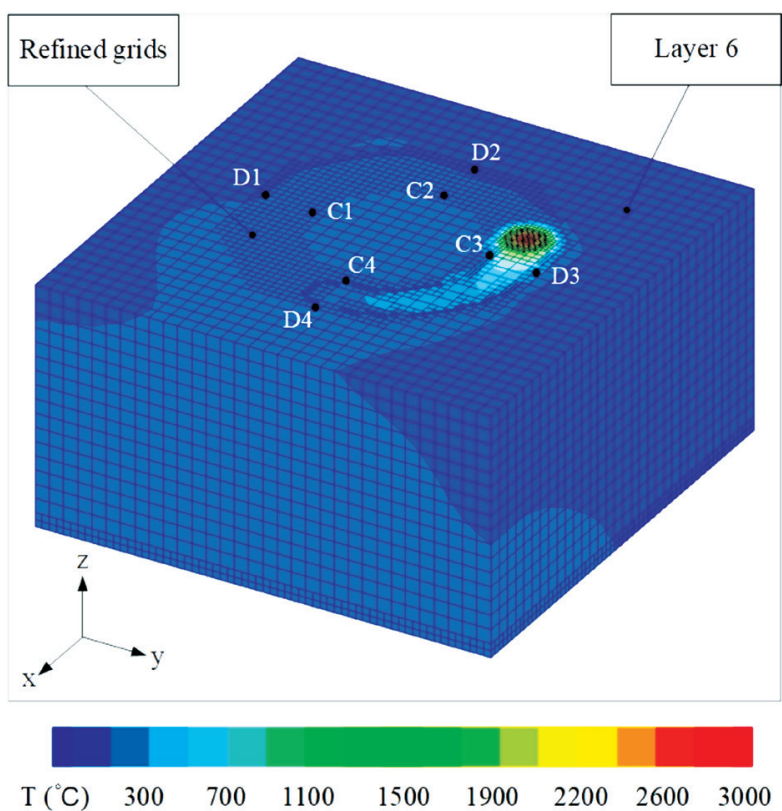

Fig. 4: Grids refining and coarsening during SLM with powder bed

computational accuracy, it also leads to larger calculations. For this reason, once the heat source goes away, the remaining grids will coarsen according to the adaptive rule. In this way, FTT functions well in temperature simulation, and it contributes greatly to the data output and simulation precision.

\subsection{Melting pool characteristic}

As shown in Fig. 6, this simulation of the impinging laser beam onto the powder bed results in the generation of a molten pool on it. The black solid line shows the dimensions of the molten pool and the black dashed line shows the dimensions of sintered region. The width of the molten pool is measured from the molten material along the direction pointing to cylinder rotation axis perpendicular to the laser scanning direction, while the melted depth is measured from the top surface of the powder bed to the molten depth along the $z$-direction as obtained on the cross section.

Figure 6 shows the temperature contour plots of specified cross section during SLM and demonstrates the molten pool. It can 

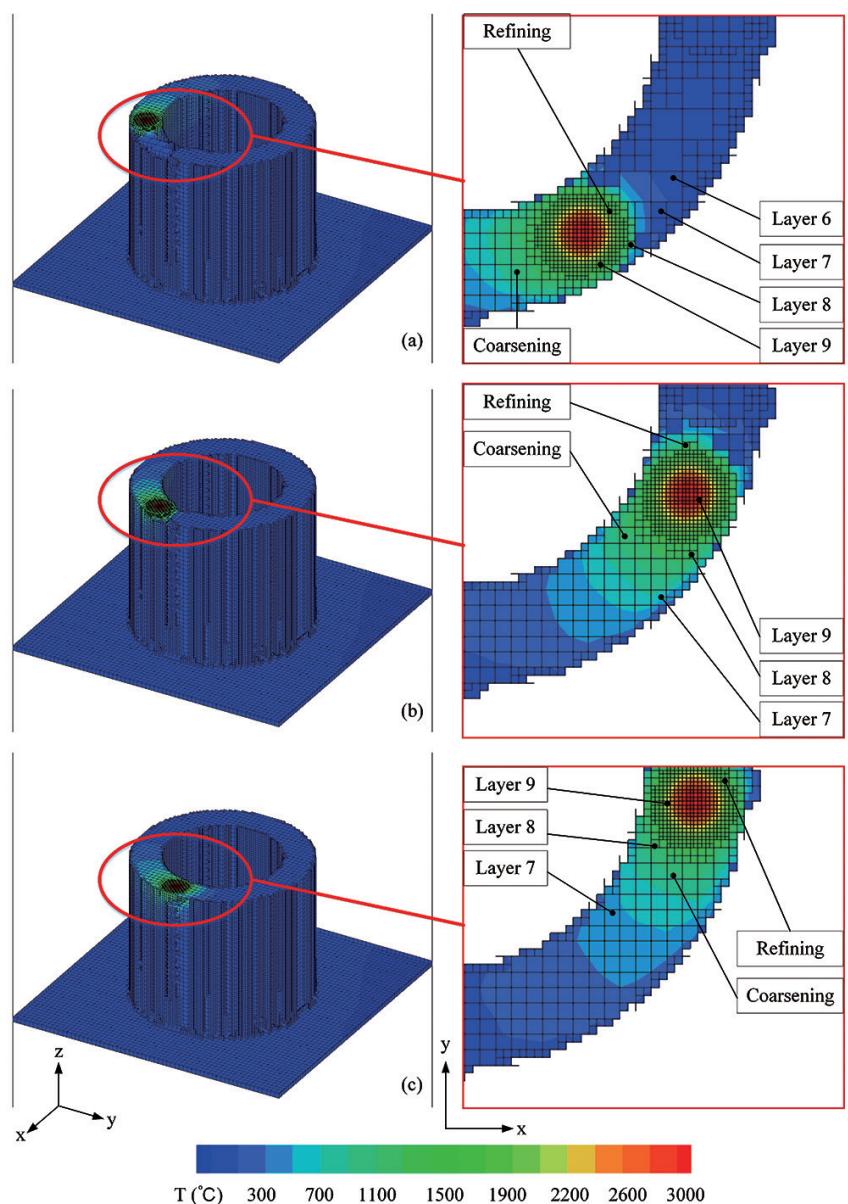

Fig. 5: Grids refining and coarsening during SLM with powder bed removed

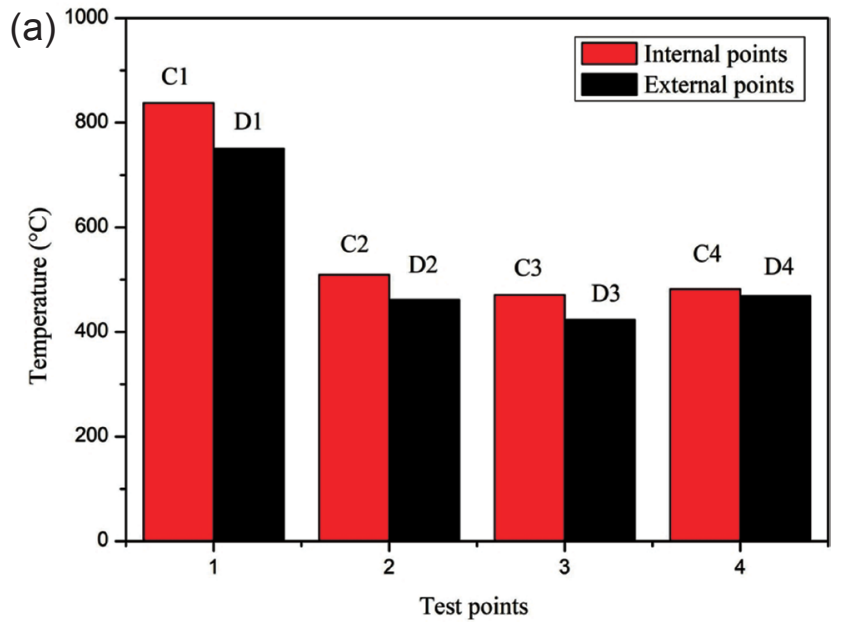

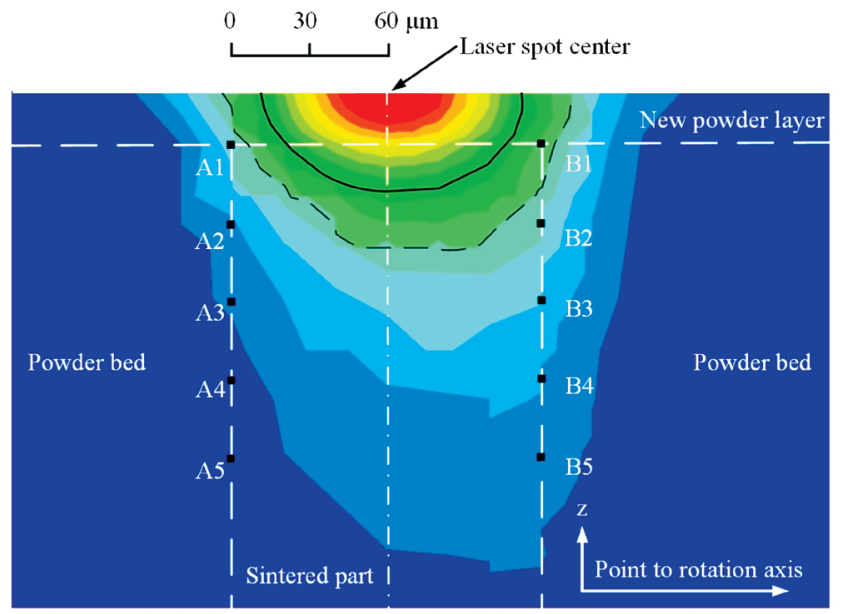

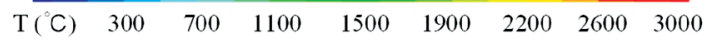

Fig. 6: Melting pool during SLM process

be seen that, instead of symmetry about the laser spot axis, the melting pool shift slightly right in Fig. 6. The right-hand side of Fig. 6 refers to the rotation axis of the cylinder in Fig. 4, pointing to the inner of the fabricated cylinder. It also can be seen that isotherm of the temperature field shift obviously right instead of symmetric about the laser spot axis. As shown in Fig. 7 (a), temperatures of the points marked in Fig. 4 are compared. These points are internal or external of cylinder and on the top surface of the powder bed. Also, as shown in Fig. 7 (b), temperatures of the points marked in Fig. 6 are compared. These points are internal or external of cylinder and along the depth of powder

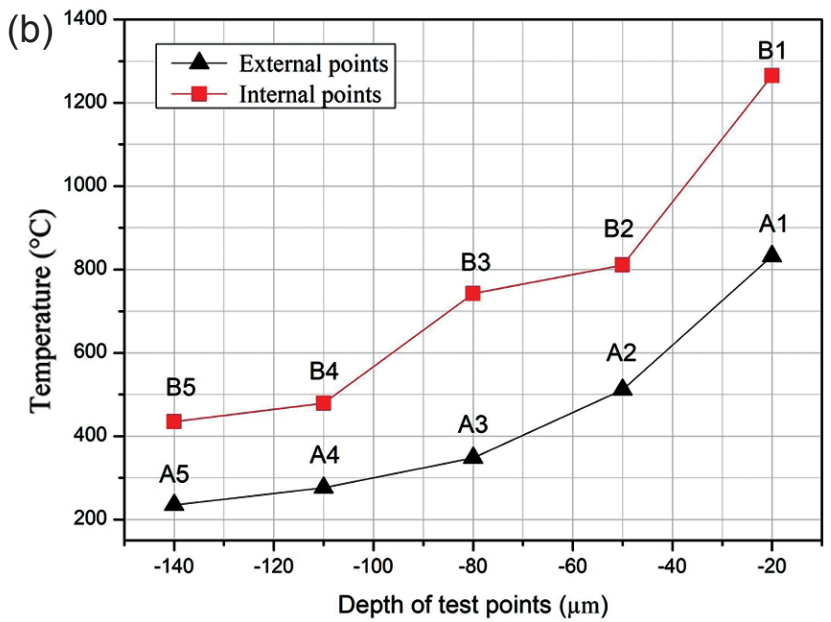

Fig. 7: Temperature of test points on surface of powder bed (a) and along depth of powder bed (b)

bed. As shown in Fig. 4 and Fig. 7 (a), for all of the four regions, the internal points temperatures are higher than the external ones. As shown in Fig. 6 and Fig. 7 (b), even if temperature decreases with the increase of depth, the internal points temperatures are about $200-400{ }^{\circ} \mathrm{C}$ higher than corresponding external ones.

It can be deduced that because the residual heat of powder bed in the inner side of cylinder could not discharge easily in conduction manner, the inner powder bed has preheating effect on fresh powder layer, and heat preservation effect on inner part of the cylinder. This results in the temperature field asymmetry and temperature gradient difference between inner and outside of cylinder. In other words, even in the same process parameters, different regions of the fabricated part have different properties during SLM. 


\section{Conclusions}

This work has applied the FTT adaptive grids technique to TC4 powders SLM temperature field simulation. The grids refining and coarsening, and the melting pool during SLM process have been investigated.

The evidence presented in this study suggests the following:

(1) Grids refining or coarsening depends on real-time temperature of a certain region. If the temperature is higher than a set value, the grids of certain region will refine, otherwise the grids will be the opposite. In the process of simulation, the grids refining in the important region ensures simulation precision, on the other hand grids coarsening in the non-key region reduces data storage and output.

(2) The inner powder bed, whose temperature is higher than external one, has preheating effect on fresh powder layer, and heat preservation effect on inner part of the cylinder. This results in the temperature field asymmetry and temperature gradient different between inner and outside of the cylinder.

\section{References}

[1] Zeng K, Pal D, Stucker B. A review of thermal analysis methods in Laser Sintering and Selective Laser Melting. In: Proceedings of Solid Freeform Fabrication Symposium Austin, TX. 2012: 796-814.

[2] Olakanmi E O, Cochrane R F, Dalgarno K W. A review on selective laser sintering/melting (SLS/SLM) of aluminium alloy powders: Processing, microstructure, and properties. Progress In Materials Science, 2015, 74: 401-477.

[3] Pengju X, Yan W, Jun H, et al. Rapidly and Effectively Forming Complex Parts with High Performance by the Compound Process of HIP and SLS/SLM. Electromachining \& Mould, 2014, 1: 014.

[4] Kolossov S, Boillat E, Glardon R E M, et al. 3D FE simulation for temperature evolution in the selective laser sintering process. International Journal of Machine Tools and Manufacture, 2004, 44(2): 117-123.

[5] Zhang D Q, Cai Q Z, Liu J H, et al. Select laser melting of W-Ni-Fe powders: simulation and experimental study. The International Journal of Advanced Manufacturing Technology, 2010, 51(5): 649-658.

[6] Wang X C, Laoui T, Bonse J, et al. Direct selective laser sintering of hard metal powders: Experimental study and simulation. International Journal of Advanced Manufacturing Technology, 2002, 19(5): 351-357.
[7] Zäh M F, Lutzmann S. Modelling and simulation of electron beam melting. Production Engineering, 2010, 4(1): 15-23.

[8] Kuchi S C. Effect of Finite Geometry on Solidification Microstructure in Beam-Based Fabrication of Thin Wall Structures. Wright State University, 2009.

[9] Sun S, Zheng L, Liu Y, et al. Selective laser melting of Al-Fe$\mathrm{V}$-Si heat-resistant aluminum alloy powder: modeling and experiments. International Journal of Advanced Manufacturing Technology, 2015, 32: 81.

[10] Fox J, Beuth J. Process mapping of transient melt pool response in wire feed e-beam additive manufacturing of Ti-6Al4V. In: Proc. Solid Freeform Fabrication Symposium, August, 2013: 675-683.

[11] Gockel J, Beuth J, Taminger K. Integrated control of solidification microstructure and melt pool dimensions in electron beam wire feed additive manufacturing of Ti-6Al-4V. Additive Manufacturing, 2014, 1: 119-126.

[12] Dai D, Gu D. Thermal behavior and densification mechanism during selective laser melting of copper matrix composites: simulation and experiments. Materials \& Design, 2014, 55: 482-491.

[13] Patil R B, Yadava V. Finite element analysis of temperature distribution in single metallic powder layer during metal laser sintering. International Journal of Machine Tools and Manufacture, 2007, 47(7): 1069-1080.

[14] Zhou W, Wang X, Hu J, et al. Melting process and mechanics on laser sintering of single layer polyamide 6 powder. The International Journal of Advanced Manufacturing Technology, 2013, 69(1-4): 901-908.

[15] Yagi S, Kunii D. Studies on effective thermal conductivities in packed beds. AIChE Journal, 1957, 3 (3): 373-381.

[16] Xue S, Barlow J W. Models for the Prediction of the Thermal Conductivities of Powders. In: Proc. Solid Freeform Fabrication Symposium, Austin, Tex.: Center for Materials Science, University of Texas at Austin, 1991: 62-69.

[17] Sih S S, Barlow J W. Measurement and prediction of the thermal conducnvtty of powders at high temperatures. Solid Freeform Fabrication, 1994: 321.

[18] Song B, Dong S, Liao H, et al. Process parameter selection for selective laser melting of Ti6Al4V based on temperature distribution simulation and experimental sintering. The International Journal of Advanced Manufacturing Technology, 2012, 61(9): 967-974.

[19] Yevko V, Park C B, Zak G, et al. Cladding formation in laserbeam fusion of metal powder. Rapid Prototyping Journal, 1998, 4(4): 168-184.

[20] Yin Y J. Research on the microstructure simulation of the alloy solidification based on phase field method by the octree mesh technology. Ph. D dissertation: Huazhong University of Science and Technology, Wuhan: 2013. (In Chinese)

This work was financially supported by the Horrizon 2020 "Efficient Manufacturing for Aerospace Components Using Additive Manufacturing, Net Shape HIP and Investment Casting (EMUSIC)", and the State Key Laboratory of Materials Processing and Die \& Mould Technology Research Project, China (2015-2017) 